\title{
Geometric Model for a Parametric Study of the Blended-Wing-Body Airplane*
}

\author{
C. Wayne Mastin ${ }^{\dagger}$ \\ Robert E. Smith ${ }^{\ddagger}$ \\ Ideen Sadrehaghighi ${ }^{\S}$ \\ Michael R. Wiese
}

\begin{abstract}
A parametric model is presented for the blendedwing-body airplane, one concept being proposed for the next generation of large subsonic transports. The model is defined in terms of a small set of parameters which facilitates analysis and optimization during the conceptual design process. The model is generated from a preliminary CAD geometry. From this geometry, airfoil cross sections are cut at selected locations and fitted with analytic curves. The airfoils are then used as boundaries for surfaces defined as the solution of partial differential equations. Both the airfoil curves and the surfaces are generated with free parameters selected to give a good representation of the original geometry. The original surface is compared with the parametric model, and solutions of the Euler equations for compressible flow are computed for both geometries. The parametric model is a good approximation of the CAD model and the computed solutions are qualitatively similar. An optimal NURBS approximation is constructed and can be used by a CAD model for further refinement or modification of the original geometry.
\end{abstract}

\footnotetext{
${ }^{*}$ This paper is declared a work of the U. S. Government and is not subjected to copyright protection in the United States.

${ }^{\dagger}$ NRC Senior Research Associate, NASA Langley Research Center, Hampton, Virginia 23681-0001.

$\ddagger$ Senior Research Engineer, NASA Langley Research Center, Hampton Virginia 23681-0001, Associate Fellow, AIAA.

${ }^{\S}$ Research Associate, Department of Mechanical Engineering, Old Dominion University, Norfolk, Virginia 23529-0247.

"Member of the Technical Staff, Computer Sciences Corporation, 3217 N. Armistead Avenue, Hampton, VA 23666.
}

\section{NOMENCLATURE}

$\begin{array}{ll}\mathbf{D}_{0}, \mathbf{D}_{1} & \text { Dirichlet boundary conditions } \\ \mathbf{N}_{0}, \mathbf{N}_{1} & \text { Neumann boundary conditions } \\ \mathbf{P} & \text { Point on NURBS surface } \\ \overline{\mathbf{P}} & \text { NURBS control points } \\ \mathbf{X} & \text { Surface coordinate } \\ C & \text { Airfoil chord length } \\ F_{1}, F_{2} & \text { Airfoil thickness parameter } \\ M_{1}, M_{2} & \text { Airfoil camber parameters } \\ N & \text { NURBS basis function } \\ P_{1}, P_{2} & \text { Airfoil camber parameters } \\ T & \text { Airfoil thickness parameter } \\ h & \text { Wing section span } \\ u, v & \text { Surface parametric variables } \\ w & \text { NURBS weight } \\ x, y, z & \text { Surface coordinates } \\ \bar{x}, \bar{y} & \text { Airfoil coordinates } \\ x_{e}, y e & \text { Airfoil trailing edge } \\ \hat{y} & \text { CAD airfoil coordinate } \\ \bar{y}_{c} & \text { Airfoil camber } \\ \bar{y}_{t} & \text { Airfoil thickness } \\ \theta & \text { Twist angle } \\ \xi, \eta & \text { Wing section parametric variables } \\ \sigma & \text { PDE weighting factor }\end{array}$

\section{INTRODUCTION}

Many of the busiest airports in Asia will run out of space in the near future. One solution to the problem proposed by the aircraft industry is the development of huge airliners carrying up to 800 passengers. Along with the tradition aircraft configurations, some manufacturers, including McDonald Douglas ${ }^{1}$, have advanced a flying wing configuration similar to the B2 bomber. This unconventional blended-wingbody (BWB) concept for future subsonic transports is an aerodynamically efficient airplane with the interior passenger volume distributed over a large portion of the centerbody. 
New concepts in aircraft designs often begin with only a rough sketch on the designers note pad. These conceptual designs always have to be modified due to structural and aerodynamic constraints. For this reason, it is convenient to have a model which is defined by a set of parameter values so the the model can be easily modified as more design variables are considered. This report will describe the application of a conceptual design tool that will facilitate aerodynamic analysis in the early stages in the development of an aircraft. The parametric model which is generated in this manner now can be optimized to improve the aerodynamic characteristics of the aircraft by varying a small set of parameters, or the complete parameter space can be examined to determine all possible feasible designs. By performing a more complete analysis at the early stages of the development, the developer will be less likely to have to make major design changes in the final Computer-Aided Design (CAD) models to be used for Computational Fluid Dynamics (CFD) analysis and wind tunnel testing.

This report will describe an application of the conceptual design tool Rapid Airplane Parametric Input Design (RAPID) $)^{2,3}$ in creating a parametric model for the the BWB airplane. Since RAPID was developed for modeling conventional airplane geometries, modifications were necessary to apply the techniques to the current application. The input parameters for a geometry may come from a variety of sources and range from crude sketches to detailed drawings or surfaces generated from a CAD system. For the BWB geometry a preliminary CAD model was available and was used to generate most of the parameters used in the RAPID model.

\section{AIRFOIL APPROXIMATION}

The construction of the parametric model for the BWB begins with the definition of airfoil cross sections. The actual CAD data was used to define the airfoil parameters in RAPID. The number of airfoils defining the model determines the total number of parameters in the model and also effects the fidelity of the model. If a large number of cross sections are made, the CAD model can be more accurately approximated by the RAPID model. However, the number of parameters increases with the number of airfoils, and there is the ever present danger of oscillations in the model that is inherent in this as well as many other interpolation and approximation schemes. From the CAD surface given in Figure 1, six cuts were made at the positions indicated in Fig- ure 2. Four of these cuts were on the main horizontal section of the wing and two were on the vertical winglet. These cross sections were output as a dense set of points. The airfoils in the parametric model were then generated with the parameters selected to give the best fit to the CAD airfoils. The parametric airfoil equations defining the coordinates $\bar{x}(\xi)$ and $\bar{y}(\xi)$ of the points on an airfoil are

$$
\begin{gathered}
\bar{x}(\xi)=C \sin \pi \xi, \quad \bar{y}(\xi)=\bar{y}_{t}(\xi)+\bar{y}_{c}(\xi), \\
\bar{y}_{t}(\xi)=-\frac{T}{2}\left(\sin 2 \pi \xi+F_{1} \sin 4 \pi \xi+F_{2} \sin 6 \pi \xi\right) \\
\bar{y}_{c}(\xi)=\left\{\begin{array}{c}
\frac{M_{1}}{P_{1}^{2}}\left(2 P_{1} r-r^{2}\right) \quad \text { if } r \leq P_{1} \\
\frac{M_{1}}{\left(P_{2}-P_{1}\right)^{3}}\left(r-P_{2}\right)^{2}\left(2 r+P_{2}-3 P_{1}\right) \\
-\frac{M_{2}}{\left(P_{2}-P_{1}\right)^{3}}\left(r-P_{1}\right)^{2}\left(2 r+P_{1}-3 P_{2}\right) \\
\text { if } P_{1}<r<P_{2} \\
\frac{M_{2}}{\left(1-P_{2}\right)^{2}}\left(1-2 P_{2}+2 P_{2} r-r^{2}\right) \quad \text { if } r \geq P_{2}, \\
r=\sin \pi \xi \\
0 \leq P_{1} \leq P_{2} \leq 1, \quad 0 \leq \xi \leq 1 .
\end{array}\right.
\end{gathered}
$$

The design parameters in these equations allow sufficient freedom to give a good approximation to all the airfoil cross sections used for the model. The parameter $C$ is the section chord length. The thickness curve $\bar{y}_{t}(\xi)$ is defined with the thickness parameter $T$ and Fourier coefficients $F_{1}$ and $F_{2}$. A generalization of the piecewise quadratic camber curve in the RAPID system has been developed to model the wing sections of the BWB configuration. The camber curve $\bar{y}_{c}(\xi)$ is a piecewise cubic curve which can have both a local maximum and a local minimum. The camber curve has relative extrema of $M_{1}$ and $M_{2}$ at locations $P_{1}$ and $P_{2}$, respectively. The camber locations are the fractions of total chord length measured from the airfoil trailing edge. If $P_{1}=P_{2}$ and $M_{1}=M_{2}$ this expression reverts to the original piecewise quadratic camber curve in RAPID. The additional parameters are needed for the BWB model to approximate the forward camber and aft reflex of the centerbody airfoil.

The selection of a set of design parameters to fit a given airfoil can be accomplished by solving a nonlinear optimization problem. Assume that a set of values $\xi_{i}, i=1, \cdots, m$ have been selected based on some desired criteria for distributing points around the airfoil. From these values, a corresponding set of values $\bar{x}_{i}, i=1, \cdots, m$ can be calculated from the first equation in (1). Now use the airfoils from the CAD data and calculate corresponding $y$ coordinates $\hat{y}_{i}, i=1, \cdots, m$, by interpolating the data if necessary. Observe that on the upper and lower surfaces 
of the airfoil the value of $\bar{y}$ can be given as a singlevalued function of $\bar{x}$. The chord length $C$ can be measured and will not be included in the set of variables for the optimization problem. Thus, the best fit to the given CAD data is the airfoil defined by equation (1) with the remaining design parameters $T, F_{1}, F_{2}, M_{1}, M_{2}, P_{1}, P_{2}$ chosen to minimize

$$
\sum_{i=1}^{m}\left[\bar{y}_{i}\left(T, F_{1}, F_{2}, M_{1}, M_{2}, P_{1}, P_{2}\right)-\hat{y}_{i}\right]^{2}
$$

The solution of this nonlinear least-squares problem defines the airfoil shapes for the parametric model. This optimization problem can be easily solved by a number of methods. The method which has been used in this work is the truncated Newton method in the TNBC package developed by S. G. Nash ${ }^{4}$. The software is designed for minimization with bound constraints and can be obtained from netlib. Other data is necessary to position the airfoils in the proper location in three dimensional space. From the airfoils in Figure 2, the spanwise locations, $h$ the locations of the trailing edges, $x_{e}$ and $y_{e}$, and the twists, $\theta$, can be determined. It is assumed that each airfoil along the fuselage and wing sections are contained in a $z=$ constant plane and that both airfoils defining the winglet are in a $y=$ constant plane. Once the airfoils are defined in space, the wing surfaces can be constructed to span the airfoils and create the airplane surface.

\section{WING SURFACES}

The RAPID methodology creates wing surfaces in $x y z$-space in the form

$$
\mathbf{X}=(x(\xi, \eta), y(\xi, \eta), z(\xi, \eta)), 0 \leq \xi \leq 1,0 \leq \eta \leq 1
$$

The coordinates of surface points are obtained by solving the fourth order partial differential equation

$$
\left[\sigma^{2} \frac{\partial^{2}}{\partial \xi^{2}}+\frac{\partial^{2}}{\partial \eta^{2}}\right]^{2} \mathbf{X}=0
$$

where $\sigma$ is a constant weighting parameter. Dirichlet and Neumann boundary conditions are imposed at $\eta=0$ and $\eta=1$. The boundary conditions are:

$$
\begin{array}{rlrl}
\mathbf{X}(\xi, 0) & =\mathbf{D}_{0}(\xi), & \mathbf{X}(\xi, 1)=\mathbf{D}_{1}(\xi), \\
\mathbf{X}_{\eta}(\xi, 0) & =\mathbf{N}_{0}(\xi), & \mathbf{X}_{\eta}(\xi, 1) & =\mathbf{N}_{1}(\xi) .
\end{array}
$$

The solution is periodic in $\xi$.

The Dirichlet boundary conditions, defined by the functions $\mathbf{D}_{0}(\xi)$ and $\mathbf{D}_{1}(\xi)$, are determined from the airfoils which form the edges of the surface. For example, if the airfoil generated in Section 2 corresponded to $\xi=0$, then the Dirichlet boundary condition would be

$$
\mathbf{D}_{0}(\xi)=\left\{\begin{array}{l}
x_{e}+\bar{x}(\xi) \cos (\theta)+\bar{y}(\xi) \sin (\theta) \\
x_{e}-\bar{x}(\xi) \sin (\theta)+\bar{y}(\xi) \cos (\theta) \\
h
\end{array}\right.
$$

The Neumann boundary conditions influence the shape of the wing surface and must be determined in part from the CAD data. From a geometric point of view, the Neumann boundary conditions can be interpreted as a tangent ribbon which defines the slope of the wing surface at the airfoil section. This tangent ribbon, as depicted in Figure 3, is constructed by offsetting the cross sections in Figure 2. For each airfoil, an offset distance in the parametric variable $\eta$ is selected. A new airfoil is constructed at the offset location using the RAPID parameters which will give a good approximation of the actual CAD cross section at the same location. Although there are a large number of parameters which determine the shape and location of an airfoil, for calculating the offset airfoil it is sufficient to consider only changes in the trailing edge, $\left(x_{e}, y_{e}\right)$, chord length, $C$, and thickness, $T$. Changes in other airfoil parameters are neglected in defining the tangent ribbon. The offset airfoils now determine the changes in $x$ and $y$ with respect to $z$, that is, $\partial x / \partial z$ and $\partial y / \partial z$ at each point. Now if $\partial z / \partial \eta$ is given, then $\partial x / \partial \eta$ and $\partial y / \partial \eta$ can be calculated from $\partial x_{e} / \partial z, \partial y_{e} / \partial z, \partial C / \partial z$, and $\partial T / \partial z$ by constructing the offset airfoil and applying the chain rule. Thus, the procedure for generating the Neumann boundary conditions can be described in the following steps: (1) calculate airfoil parameters for the offset airfoils with chord, thickness, and trailing edge coordinates given by

$$
\begin{array}{cc}
C+\frac{\partial C}{\partial z} \frac{\partial z}{\partial \eta}, & T+\frac{\partial T}{\partial z} \frac{\partial z}{\partial \eta} \\
x_{e}+\frac{\partial x_{e}}{\partial z} \frac{\partial z}{\partial \eta}, & y_{e}+\frac{\partial y_{e}}{\partial z} \frac{\partial z}{\partial \eta}
\end{array}
$$

resectively; (2) construct the offset airfoil with coordinates $\left(x_{o}(\xi), y_{o}(\xi)\right)$; and $(3)$ set the Neumann boundary conditions

$$
\mathbf{N}_{0}(\xi)=\left\{\begin{array}{l}
x_{o}(\xi)-x(\xi, 0) \\
y_{o}(\xi)-y(\xi, 0) \\
\frac{\partial z}{\partial \eta}
\end{array}\right.
$$

The spanwise changes in trailing edge coordinates, chord length and thickness can be easily estimated from the CAD data. However, an estimate of $\partial z / \partial \eta$, which gives the spanwise influence of the Neumann 
boundary condition, is not apparent from the data. Thus some trial and error was necessary to obtain the best fit to the CAD surface. In most cases, setting $\partial z / \partial \eta$ equal to the span of the wing section is a good starting value.

Once the boundary conditions are determined, equation (2) is solved by generating a Fourier series expansion. An approximation of the CAD surface using the RAPID technology appears in Figure 4.

The total number of parameters will now be summarized. There are a total of six airfoils defining the BWB geometry. Each airfoil is defined by eight parameters

$$
C, T, F_{1}, F_{2}, M_{1}, M_{2}, P_{1} \text {, and } P_{2} \text {. }
$$

The location and orientation of each airfoil in space must be defined. This is done by specifying the spanwise location of the airfoil, $h$, the coordinates of the trailing edge, $x_{e}$ and $y_{e}$, and the twist angle, $\theta$. There are additional parameters for each airfoil used to define the Neumann boundary conditions. These are

$$
\frac{\partial z}{\partial \eta}, \frac{\partial x_{e}}{\partial z}, \frac{\partial y_{e}}{\partial z}, \frac{\partial C}{\partial z} \text {, and } \frac{\partial T}{\partial z} .
$$

Thus, there are a total of 17 parameters for each of six airfoils. Now deleting the spanwise location of the centerbody airfoil which we assume to be at $z=0$, the BWB airplane, as defined by RAPID, is generated with a total of 101 parameters.

Since considerable effort has gone into attempting to approximate a given CAD surface with a parametric model, some comparisons of the original CAD surface and the RAPID surface will be presented. It is evident from comparing Figures 1 and 4 that the gross geometry of the BWB airplane has been captured in the RAPID model. Figures 5 and 6 compare the models at two cross sections used in defining the RAPID model. The approximation of the supercritical airfoil section in Figure 6 is excellent, but there is noticeably more error in the approximation of the centerbody airfoil in Figure 5. A comparison of the planforms appears in Figure 7. Whether this degree of fidelity in the approximation is sufficient would of course be a judgement call of the designer. Further accuracy could be achieved with the RAPID technology, but at the cost of increasing the complexity of the model and the number of parameters.

\section{CFD ANALYSIS}

The RAPID system can output surface grids with specified grid point distributions in each coordinate direction. These surface grids can be used directly in a potential flow analysis code or they can be used as boundary surfaces to generate a volume grid for an Euler or Navier-Stokes calculation. Similar volume grids were constructed for the region about the CAD and RAPID models. In order to guarantee comparable grids, a grid was first generated about the RAPID surface and then this grid was distorted to generate the grid about the CAD surface. The grid distortion process was carried out as a application of the Coordinate and Sensitivity Multidisciplinary Design and Optimization (CSCMDO) system ${ }^{5}$. With this system, the differences in the volume grids is no greater than the differences in the surface grids. The CSCMDO system could also be used to generate new volume grids after making small changes in design parameters. Solutions to the Euler equations for compressible flow were computed on grids for the RAPID and CAD geometries. The following comparisons are for a free stream Mach number of 0.85 and an angle of attack of 2 degrees. Figure 8 contains comparisons of contour plots of the local Mach number on the upper surfaces of the CAD and RAPID geometries. The similarity in the solutions is evident, especially the shock structure along the wing segment. Further comparisons reveal the effects of the differences in geometry. Figures 9 and 10 are pressure coefficient plots near the center of the fuselage cross section and on the wing. The pressure coefficient plots are superimposed over plot of the airfoil cross sections, so that difference in solutions can be compared with the differences in geometries. It was interesting to note that the RAPID surface geometry gave smoother plots and a larger lift coefficient than the solution computed with the CAD geometry.

\section{NURBS APPROXIMATION}

NonUniform Rational B-Splines (NURBS) have become a standard surface representation in the CAD industry ${ }^{6}$. For this reason, a method for accurately representing the RAPID surface geometry by a NURBS surface has been investigated. As with the RAPID methodology, one of the advantages in a NURBS representation of a surface is the fact that the surface is defined by a set of parameters which can be manipulated to change the shape of the surface. However, there is a difference in the two representations. The RAPID parameters are actual geometric lengths and distances, whereas the 
NURBS parameters are the control points, weights, and knots.

A NURBS surface of degree $p$ in the $u$-direction and degree $q$ in the $v$-direction is a piecewise rational function of the form

$$
\mathbf{P}(u, v)=\frac{\sum_{i=0}^{I} \sum_{j=0}^{J} N_{i, p}(u) N_{j, q}(v) w_{i, j} \overline{\mathbf{P}}_{i, j}}{\sum_{i=0}^{I} \sum_{j=0}^{J} N_{i, p}(u) N_{j, q}(v) w_{i, j}}
$$

where $\overline{\mathbf{P}}_{i, j}$ are the points of the control net, $w_{i, j}$ are the weights, and $N_{i, p}(u)$ and $N_{j, q}(v)$ are the Bspline basis functions defined on the knot vectors $\bar{u}_{0}, \cdots, \bar{u}_{I+p+1}$ and $\bar{v}_{0}, \cdots, \bar{v}_{J+q+1}$ with

$$
\begin{gathered}
\bar{u}_{0}=\cdots=\bar{u}_{p}=0=\bar{v}_{0}=\cdots=\bar{v}_{q+1} \\
\bar{u}_{I+1}=\cdots=\bar{u}_{I+p+1}=1=\bar{v}_{J+1}=\cdots=\bar{v}_{J+q+1} .
\end{gathered}
$$

Let $\mathbf{X}_{m, n}, m=1, \cdots, M, n=1, \cdots, N$ be a surface grid generated by RAPID. Surfaces as NURBS are defined on the unit square and can be evaluated at an array of points $\left(u_{m}, v_{n}\right), m=1, \cdots, M, n=$ $1, \cdots, N$ in the $u v$-plane. The optimal NURBS approximation is obtained by selecting the control net, weights, and knots which yields the minimum value of

$$
\sum_{m=1}^{M} \sum_{n=1}^{N}\left[\mathbf{X}_{m, n}-\mathbf{P}\left(u_{m}, v_{n}\right)\right]^{2} .
$$

The optimal NURBS will depend on the selection of the array of parametric variables $u$ and $v$. Both uniform and arclength parameters have worked well in practice.

An example of a NURBS approximation of the BWB airplane and the control net is plotted in Figure 11. This surface is defined using piecewise cubic basis functions in both directions and an $8 \times 8$ control net. The NURBS surface is therefore defined by 64 values for each coordinate of the control net, 64 weights, and four knot values for each direction. Thus the NURBS surface is defined with a total of 264 parameters.

The NURBS approximation requires the solution of a nonlinear optimization problem. The solution is computed using a two-stage process. First, the optimal control net is constructed using unit weights and uniform knots. This is a linear least-squares problem that is solved directly using a QR matrix factorization. The resulting set of control points with unit weights and uniform knots are initial values for calculation of the optimal NURBS. Next, the full nonlinear problem with variable control net, weights, and knots is solved using the TNBC optimization routine.

\section{SUMMARY AND CONCLUSIONS}

The RAPID methodology has been used to generate a parametric model for the BWB subsonic transport. With only a small set of parameters, there is a limit to the accuracy in the approximation of the original CAD model. In examining the differences in geometry and CFD solutions with the CAD and Rapid models, it should always be noted that this methodology is intended for aerodynamic analysis at the conceptual stage of the design process. At this stage, the CAD geometry may only be a crude approximation of the final configuration. The objective in constructing the RAPID model is to have a parametrization of the BWB configuration. The RAPID model can then be used in a parameter study to predict the effects of changes in the geometry and improve upon the original design.

\section{ACKNOWLEDGEMENTS}

This work was performed while the first author held a National Research Council-NASA Langley Research Center Research Associateship.

\section{References}

${ }^{1}$ Liebeck, R. H., Page, M. A., and Rawdon, B. K., "Evolution of the Revolutionary Blended-Wing-Body," Transportation Beyond 2000: Technologies Needed for Engineering Design, NASA CP 10184, pp 431-459, 1996.

${ }^{2}$ Smith, R. E., Bloor, M. G. I., Wilson, M. J., and Thomas, A. M., "Rapid Airplane Parametric Input Design (RAPID)," AIAA Paper 95-1687, June, 1995.

${ }^{3}$ Smith, R. E., Cordero, Y., and Jones, W. T., "Automated Airplane Surface Generation," Numerical Grid Generation in Computational Field Simulations pp. 293-301, NSF Engineering Research Center for Computational Field Simulation, Mississippi State, MS, 1996.

${ }^{4}$ Nash, S. G., User's guide for TN/TNBC, Technical Report 397, Department of Mathematical Sciences, The Johns Hopkins University, Baltimore, MD, 1984.

5 Jones, W., and Samareh-Abolhassani, J., "A Grid Generation System for Multi-disciplinary Design," AIAA Paper 95-1689, June, 1995.

${ }^{6}$ Tiller, W., "Rational B-Splines for Curve and Surface Representation," IEEE Computer Graphics and Applications, 3, pp. 61-69, 1983. 


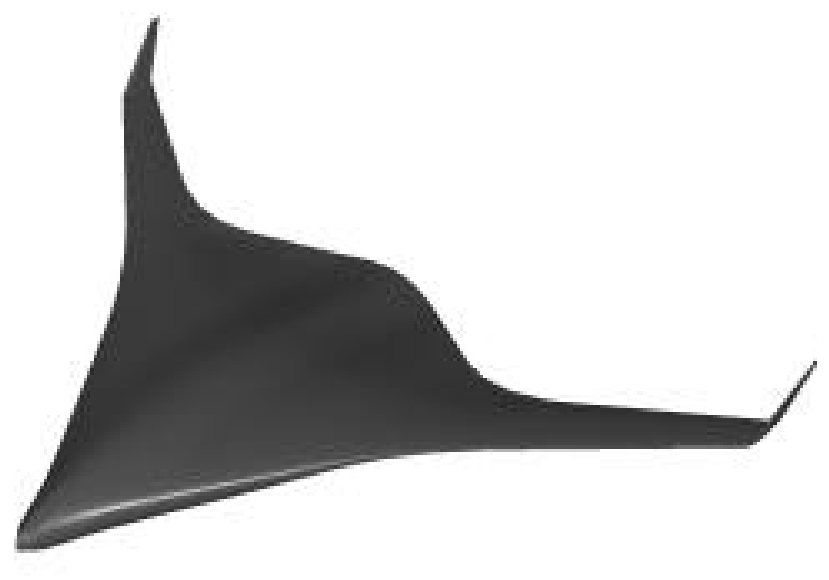

Fig. 1 Blended-wing-body CAD geometry
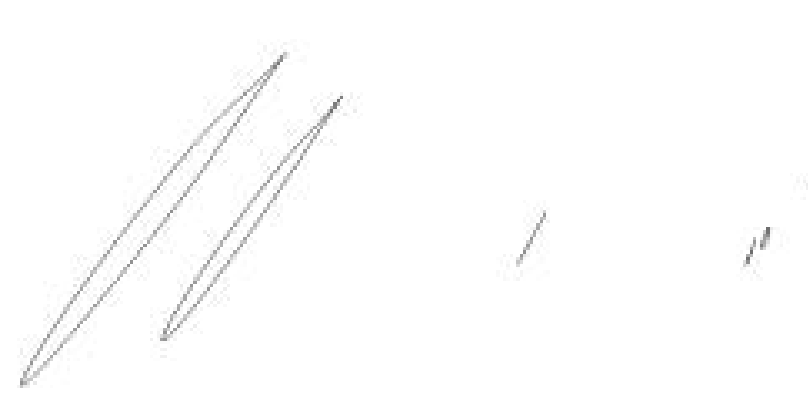

Fig. 2 Airfoil cross sections for RAPID model

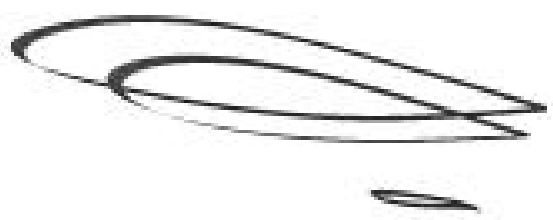

Fig. 3 Tangent ribbons for RAPID model

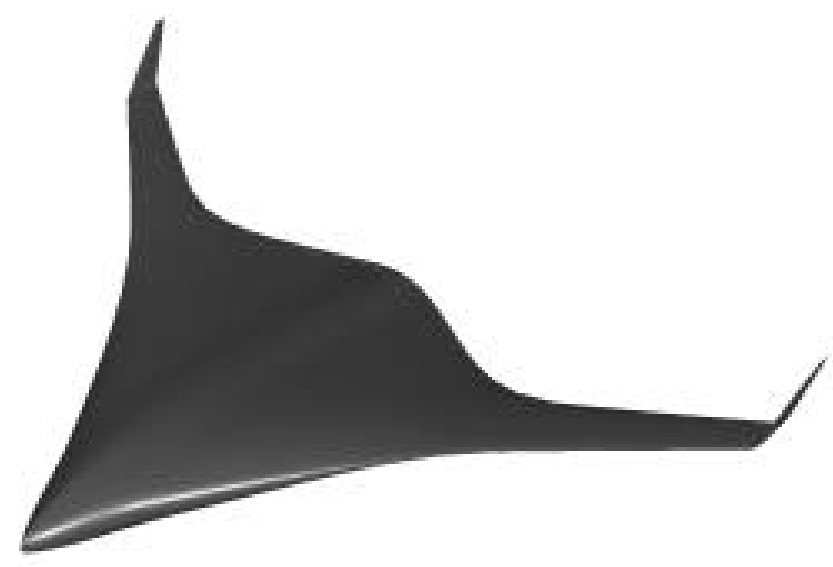

Fig. 4 Blended-wing-body RAPID geometry 


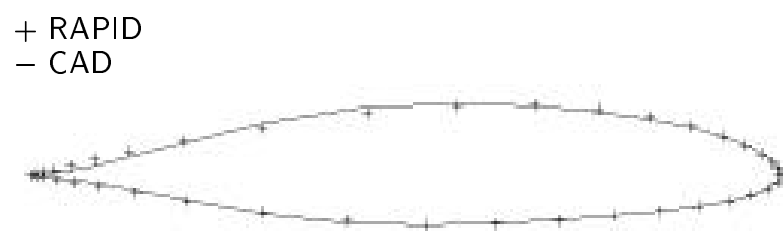

Fig. 5 Comparison of CAD and RAPID model for center body airfoil

+ RAPID

- CAD

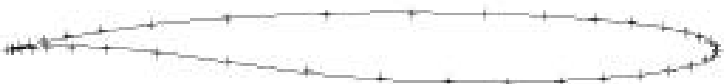

Fig. 6 Comparison of CAD and RAPID model for wing airfoil

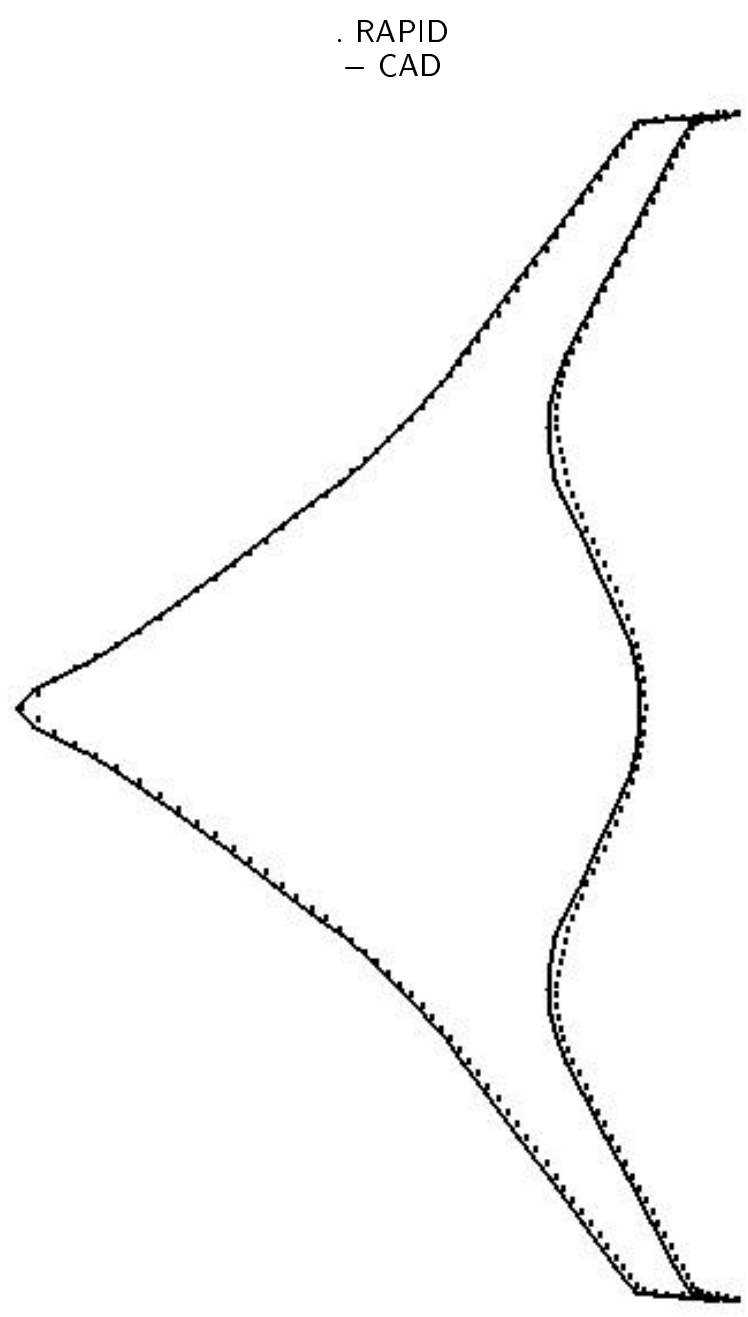

Fig. 7 Comparison of CAD and RAPID planforms 


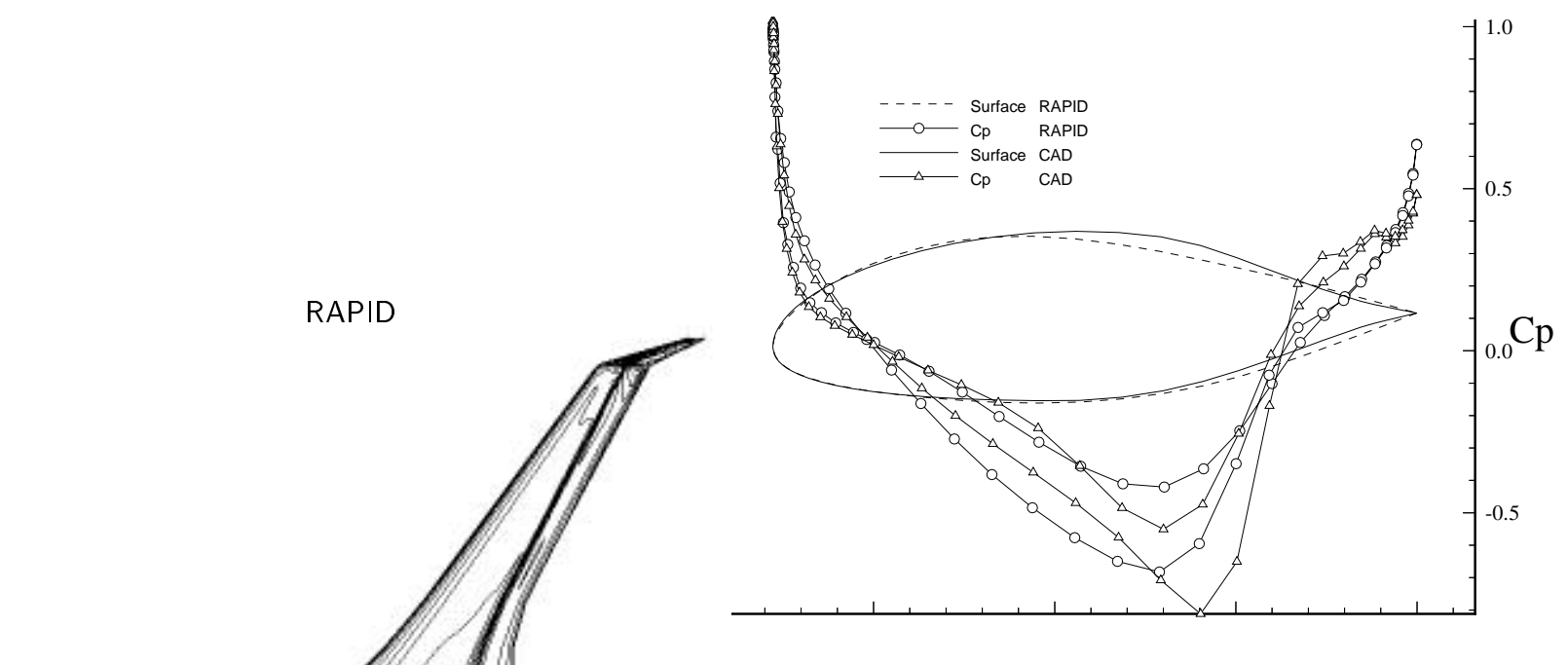

Fig. 9 Pressure coefficient at fuselage cross section

CAD

Fig. 8 Mach contours of CAD and RAPID models

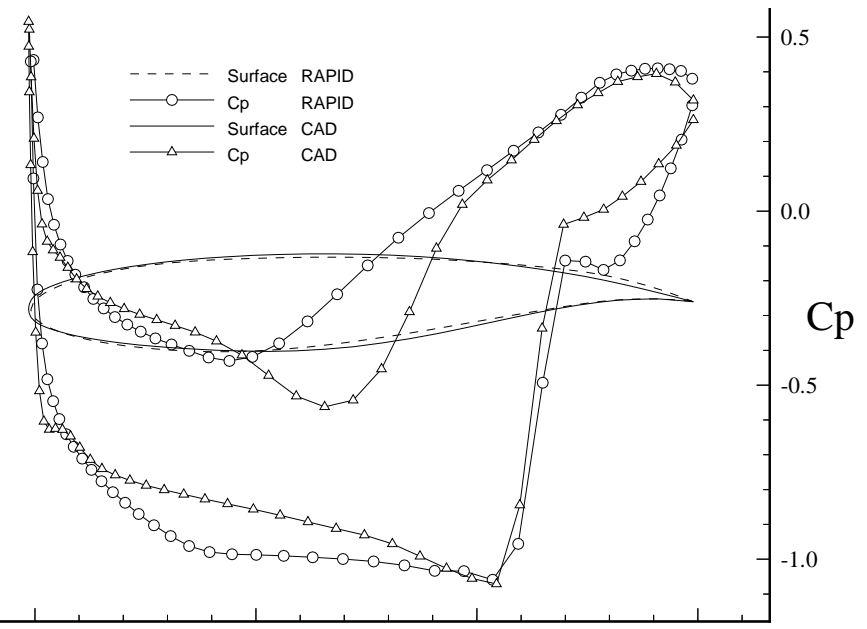

Fig. 10 Pressure coefficient at wing cross section 


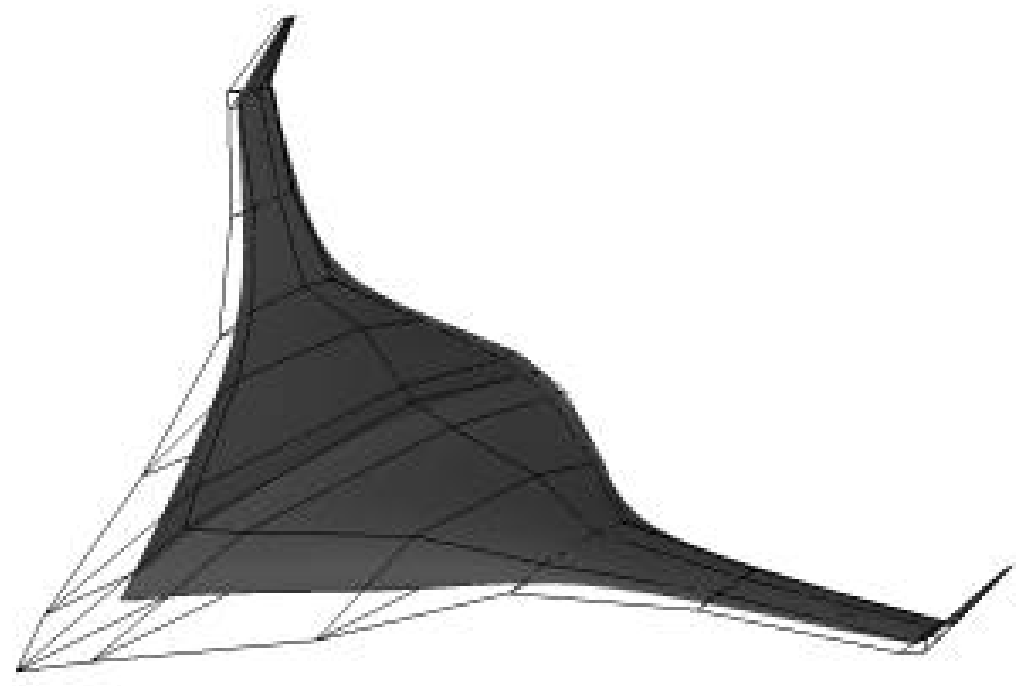

Fig. 11 NURBS approximation of RAPID geometry 